\title{
Plant Biotechnology in the Field-A Snapshot with Emphasis on Horticultural Crops
}

\author{
Rebecca Grumet \\ Department of Horticulture, Michigan State University, East Lansing, MI 48824
}

Within a relatively short time, the plant biotechnology industry has progressed from the first small-scale field trials in 1987 to the commercial release of transgenic crops in the mid- to late 1990s. A tremendous portion of the U.S. acreage was committed to genetically engineered cultivars of major agronomic crops within 3 to 4 years of commercial release of the first wave of transgenic crops. Acreage of cotton planted to transgenic cultivars grew rapidly from $10 \%$ in 1997 , to $26 \%$ in 1998 , and $46 \%$ in 2000 , while acres of transgenic soybean increased from $15 \%$ in 1997 to $>50 \%$ in 2000 [Brownlee, 1998; Meyerson, 1997; Economic Research Service, USDA (http:// www.ers.usda.gov/)]. Although transgenic horticultural crops have not achieved the same level of production, several transgenic products have reached the market, and a new generation of engineered horticultural crops is likely to follow in the next decade.

This brief overview provides a snapshot of the commercial or near-commercial production of transgenic horticultural crops. The U.S. Dept. of Agriculture-Animal and Plant Health Inspection Service (USDA-APHIS) records of field trials of genetically engineered plants have been a valuable source of information in compiling this report. Since experimental field trials with transgenic plants must be registered, this information is available through the USDAAPHIS-BSS (Biotechnology and Scientific Services, "BM1," (http://www.aphis.usda.gov/ ppq/biotech/) and the Virginia Tech, Information Systems for Biotechnology (ISB) (http:// www.nbiap.vt.edu/cfdocs/fieldtests.1.cfm), websites.

The initial transgenic products relied heavily on cloning genes based on well-understood biochemical or physiological systems, or on utilizing genes from organisms that are comparatively easy to manipulate (e.g., viruses or bacteria). For example, altered ripening characteristics took advantage of welldefined, postharvest physiology processes with regard to ethylene production and enzymes associated with cell wall softening; Bt (Bacillus thurengiensis)-based insect resistances took advantage of long-known and well-understood Bt biology; herbicide resistance took advantage of known herbicide-resistant plant mutants, or the ability to screen for bacteria that can degrade a given herbicide; and engineered virus resistance utilized genes derived from viral genomes (Grumet and Gifford, 1998). These four categories of engineered traits alone account for nearly $90 \%$ of the 60 cultivars that have made it to the stage of deregulation or

Received for publication 14 Mar. 2002. Accepted 27 Mar. 2002. I thank Dr. Jim Hancock for helpful suggestions on the manuscript. application for deregulation status from the USDA as of Oct. 2001 (Table 1A). Deregulation status refers to permission from the USDA to grow at commercial levels rather than limited experimental field trials. It should be noted that before commercial production can occur, it may also be necessary to obtain approval from the FDA (Food and Drug Administration) and/or the EPA (Environmental Protection Agency).

A different picture emerges when we look at notifications for field trials rather than deregulated crops (Table 1B). More than 100 notifications were submitted in May 2001 alone. Although the two biggest categories from the deregulated materials (herbicide resistance and insect resistance) are still at the top of the list, there is now a much broader array of traits, indicating that new genes and traits are actively being developed. Examples include engineered fungal or bacterial resistance, and modified protein, oil, carbohydrates, or cell wall composition. The majority of research done to modify product quality parameters has been done on major field crops such as corn and soybeans by large companies (Brownlee, 1998).

Of the crop species that have been tested in the field, the greatest number have been with corn; more than 3200 field trials were performed from 1987 to 2001 (Table 2A). Potato, soybean, tomato, and cotton make up the next group, with 400-700 trials each. These species reflect both the interests of industry, as well as the ease of transformation. Tomato and potato are relatively easy to transform, while

Table 1. Types of traits tested in U.S. field trials of genetically engineered crop plants.

\begin{tabular}{lrrlr}
\hline \hline \multicolumn{2}{l}{ A. Deregulated by USDA as of 10/01 } & \multicolumn{2}{l}{ B. Notifications submitted 5/01-6/01 } \\
\hline Herbicide resistance & 24 & $49 \%$ & Herbicide resistance & 30 \\
Insect resistance & 16 & $26 \%$ & Insect resistance & 14 \\
Male sterility & 7 & $12 \%$ & Fungal/bacterial resistance & 12 \\
Altered ripening & 6 & $10 \%$ & Altered oil & 8 \\
Virus resistance & 5 & $8 \%$ & Altered growth/yield & 8 \\
Altered oil & 2 & $3 \%$ & Secondary metabolism & 8 \\
& 60 & & Altered carbohydrate/walls & 7 \\
Total & & & Virus resistance & 6 \\
& & & Stress resistance & 4 \\
& & & Fertility/sterility & 3 \\
& & & Altered ripening & 2 \\
\cline { 3 - 4 } & & & Total & 101 \\
\hline
\end{tabular}

Table 2. Crop species tested in transgenic field trials in the United States, 1987-2001

\begin{tabular}{|c|c|c|c|c|c|}
\hline \multicolumn{6}{|c|}{ A. Most frequent releases } \\
\hline Species & No. trials & Species & No. trials & Species & No. trials \\
\hline$\overline{\text { Corn }}$ & 3253 & Melon & 130 & Strawberry & 40 \\
\hline Potato & 695 & Sugar beet & 126 & Grape & 32 \\
\hline Soybean & 571 & Rice & 125 & Sugar cane & 31 \\
\hline Tomato & 504 & Alfalfa & 102 & Sunflower & 30 \\
\hline Cotton & 491 & Bentgrass & 97 & Apple & 28 \\
\hline Wheat & 212 & Lettuce & 69 & Barley & 28 \\
\hline Tobacco & 189 & Squash & 60 & Cucumber & 24 \\
\hline \multirow[t]{2}{*}{ Rapeseed } & 162 & Poplar & 56 & Pea & 22 \\
\hline & & & & Peanut & 20 \\
\hline
\end{tabular}

B. Fewer than 20 field releases

\begin{tabular}{lclrlrr}
\cline { 1 - 2 } Species & No. trials & & Species & No. trials & Species & No. trials \\
\cline { 1 - 3 } Amelanchier laevis & 1 & & Gladiolus & 2 & Petunia & 13 \\
Belladona & 6 & & Grapefruit & 5 & Plum & 3 \\
Bermuda grass & 5 & & Kentucky bluegrass & 18 & Pine & 15 \\
Brassica oleracea & 18 & Oat & 1 & Pineapple & 1 \\
Carrot & 13 & Onion & 4 & Raspberry & 9 \\
Cassava & 1 & Papaya & 13 & Rhododendron & 3 \\
Chrysanthemum & 2 & Pear & 5 & Spruce & 1 \\
Chicory & 1 & Pelargonium & 5 & St. Augustine grass & 2 \\
Coffee & 3 & Pepper & 12 & Sweetgum & 14 \\
Cranberry & 1 & Peppermint & 1 & Sweetpotato & 8 \\
Eggplant & 7 & Perennial ryegrass & 2 & Walnut & 12 \\
Festuca arundinacea & 7 & Persimmon & 4 & Watermelon & 9 \\
\hline
\end{tabular}


corn and soybean are difficult to transform. However, due to the large acreage and production potential, corn and soybean have benefited from intensive efforts by many groups, especially in the private sector. When dealing with horticultural crops, many species are either not easy to transform (there are exceptions) or are not of large enough commercial value to attract a great deal of attention. It can be seen, however, that after the most frequently tested group of crops, there are a variety of other species, and the list of crops that has been field tested has increased a great deal in the past few years to include numerous horticultural species. An even greater variety of horticultural crops are represented among those field tested $<20$ times (Table 2B). These trends suggest that the future should include a much greater variety of engineered crops, although many horticultural species may still lag behind.

Some limitations with regard to production of transgenic horticultural crops stem from the nature of the horticultural industry that encompasses a broad array of crops and species. Similar to the problems encountered with obtaining pesticide registration for minor crops, the transformation of numerous species means there are more species to work on, less effort invested in each, and less acreage upon which to obtain a return on investment. Since the great majority of transformation systems are dependent on regeneration from tissue culture, the appropriate conditions must be established individually for each species of interest (Birch, 1997). Furthermore, in many cases the genotypes that are most readily transformed, may not be the ones most desirable for horticultural and production characteristics. This potential limitation must then be addressed either by costly further development of regeneration and transformation systems, or by transfer of the introduced gene from a transformable genotype into a more desirable genotype by conventional breeding. The ability to achieve high frequency transformation is especially important from the standpoint of gene expression, which can be a limiting factor in achieving the desired phenotype (Birch, 1997). Both the site of integration and the number of genes integrated can influence the level of expression. Typically, large numbers of transgenic individuals must be produced with the desired gene in order to obtain lines that display the necessary levels of gene expression.

Finally, in addition to the ability to achieve successful transformation and adequate stable gene expression, there is the important question of overall performance of the new cultivar. Developing a commercially viable product requires more than cloning the right gene and finding a way to insert it. Once this is achieved, the resultant transgenic crop faces all of the issues associated with the commercial release of any new cultivar (Grumet and Gifford, 1998). Growers will judge usefulness based on performance in the field, and processors or consumers will be concerned with the suitability for their needs. After the initial small plot trials, there is the necessary stage of multiple year, multiple location trials, as for any cultivar to be released.

In fact, field performance that is not necessarily related to the engineered trait has turned out to be a tough test for some of the first wave of commercial transgenic products. For example, commercial success of the first genetically engineered crop to reach the market, the 'Flavr Savr' tomato, was ultimately hindered by difficulties in the production and distribution process (Kidd, 1995). 'Bollguard' cotton released by Monsanto received negative publicity due to a possible tendency to drop bolls more readily (Fox, 1997; Meyerson, 1997). Overall, this emphasizes the importance for molecular biologists to work closely with plant breeders and horticulturists to develop lines that will ultimately be of commercial value.

Thus many factors, from gene, to genotype, to production environment, play a role when attempting to move from the model system or test case in the laboratory to a horticulturally useful cultivar. Given the effort that is required for product development, it is perhaps not surprising to note that if we look at crops that have reached the deregulation state (Table 1A), there are only two examples (transgenic papaya engineered for resistance to papaya ringspot virus from Cornell Univ., and sulfonylurea-resistant flax from the Univ. of Saskatchewan) that were developed at universities (USDA-APHIS records). All of the other examples come from the private sector. This parallels the trend for most of the breeding and cultivar development that is being done today, regardless of whether it involves transgenic materials. Due to the high expenses involved, and the increasingly important role of various nontechnical issues such as intellectual property rights (Barton, 1995), the role of the private sector is likely to be even greater for transgenic cultivars than it has been for conventional breeding. Even in the case of the Cornell papaya, it was necessary to obtain licenses from other institutions and companies such as MIT and Monsanto (Nature Biotechnology, 1998, 16:499).

In summary, although only a limited number of transgenic crops have reached commercialization to date, the future is likely to see great increases. There was a dramatic jump in the number of field trials being performed in the late 1990s, with an average of 590 trials per year in 1994-97, vs. an average of 38 per year in the first 5 years of testing, 1992-97. The period from 1998 to 2001 showed a higher average of 1030 per year (USDA-APHIS records). The most recent trials include an increasingly broader array of both species and traits, making it very likely that there will be a more diverse range of transgenic products in the future, and that they will have a direct impact on production of horticultural crops.

\section{Literature Cited}

Barton, J.H. 1995. Patent scope in biotechnology. Intl. Rev. Property Copyright Law 26:605-618.

Birch, R.G. 1997. Plant transformation: Problems and strategies for practical application. Annu. Rev. Plant Physiol. Plant Mol. Biol. 48:297-326.

Brownlee, S. 1998. Dollars for DNA. Biotech finally seems near to living up to its hype. U.S. News and World Rpt. 25 May. p. 48-50.

Fox, J.L. 1997. Farmers say Monsanto's engineered cotton drops bolls. Nature Biotechnol. 15:1233.

Grumet, R. and F. Gifford. 1998. Plant biotechnology in the United States: Issues and challenges en route to commercial production. HortScience 33:187-192.

Kidd, G. 1995. Low-tech woes derail Calgene's high-tech tomato. Bio/Technology 13:540-541.

Meyerson, A.R. 1997. Breeding seeds of discontent. New York Times. 17 Nov. 